\title{
REDSHIFTS FOR A SAMPLE OF RADIO-SELECTED POOR CLUSTERS
}

\author{
Neal A. Miller \\ Laboratory for Astronomy and Solar Physics, Code 681, NASA Goddard Space Flight Center, Greenbelt, MD 20771; \\ nmiller@stis.gsfc.nasa.gov \\ Michael J. Ledlow \\ Gemini Observatory Southern Operations Center, clo AURA, Inc., Casilla 603, La Serena, Chile; mledlow@ gemini.edu
}

\section{Frazer N. Owen}

National Radio Astronomy Observatory, 'P.O. Box O. Socorro, NM 87801 ; fowen@aoc.nrao.edu

\author{
AND
}

JOHN M. HILL

Steward Observatory, University of Arizona, 933 North Cherry Avenue, Tucson, AZ 85721-0065; jhill@ as.arizona.edu

Received 2002 January 11; accepted 2002 March 14

\section{ABSTRACT}

Multifiber optical spectroscopy has been performed on galaxies in the vicinity of strong, nearby radio galaxies. These radio galaxies were selected from the 3CR and B2 catalogs based on their exclusion from the Abell catalog, which is puzzling given the hypothesis that an external medium is required to confine the radio plasma of such galaxies. Velocities derived from the spectra were used to confirm the existence of groups and poor clusters in the fields of most of the radio galaxies. We find that all radio galaxies with classical Fanaroff-Riley type I morphologies prove to reside in clusters, whereas the other radio galaxies often appear to be recent galaxy-galaxy mergers in regions of low galaxy density. These findings confirm the earlier result that the existence of extended X-ray emission combined with a statistical excess of neighboring galaxies can be used to identify poor clusters associated with radio galaxies.

Key words: galaxies: active-galaxies: clusters: general-galaxies: distances and redshifts

' The National Radio Astronomy Observatory is a facility of the National Science Foundation operated under cooperative agreement by Associated Universities, Inc.

\section{INTRODUCTION}

Some of the more spectacular extragalactic sources are the powerful radio galaxies, with $L_{1.4 \mathrm{GHz}} \geq$ $10^{23} \mathrm{~W} \mathrm{~Hz}^{-1}$. Associated almost exclusively with massive elliptical galaxies, these objects often exhibit radio emission extending far beyond the optical extent of the host galaxies. The more extreme examples span over $1 \mathrm{Mpc}$ with the radio emission resembling jets or lobes. In order to explain these large sizes, 
models require an external medium to confine the radio plasma and associated magnetic fields, and consequently the cluster environment, with its intracluster medium of hot gas, is an attractive candidate. Indeed, many powerful radio galaxies are found to lie in clusters. However, not all powerful radio galaxies reside in known clusters of galaxies. How can such sources be explained?

One possible explanation is that such radio galaxies do, in fact, reside in clusters of galaxies but that these clusters are simply too poor to have been included in classical catalogs. For example, the lowest-richness clusters in the Abell catalog (Abell 1958; Abell, Corwin, \& Olowin 1989) must contain at least 35 galaxies in the interval described by the cluster's third-brightest galaxy to a galaxy 2 mag fainter than this galaxy. This arbitrary cutoff implies that systems that represent meaningful associations of galaxies could easily be missed, despite having environments nearly identical to those of the poorer clusters of the catalog.

In addition, the connection between powerful radio galaxies and real galaxy overdensities might be exploited to identify samples of clusters and groups. The powerful radio galaxies would be nearly ideal signposts, as they are observable out to very high redshifts and are nearly always found in the centermost regions of clusters (Ledlow \& Owen 1995). In fact, studies, including Allington-Smith et al. (1993), have taken advantage of this technique. However, the picture is likely more complicated. Specifically, there is evidence that different types of powerful radio sources inhabit different environments. For example, radio galaxies are often separated based on radio luminosity and morphology (and optical magnitude; see Owen \& White 1991; Ledlow \& Owen 1996) into FR I and FR II sources (Fanaroff \& Riley 1974). The FR I sources appear to be more frequently associated with cluster environments than do FR II sources (see, e.g., Longair \& Seldner 1979; Miller et al. 1999), although this may only be true at lower redshift, as at higher redshift their environments appear more similar (e.g., Prestage \& Peacock 1988). There maly also be environmental differences among the more powerful radio galaxies (generally FR II), with some being fairly normal optically luminous elliptical galaxies whereas others are clearly disturbed and apparent galaxy-galaxy merger systems (Heckman et al. 1986). Consequently, in order to use radio galaxies to identify samples of clusters and groups, it appears to be important to consider the nature of the radio source.

In Miller et al. (1999, hereafter Paper I), we explored these themes using a sample of nearby powerful radio galaxies that were not members of Abell clusters. The local environments of the radio galaxies were examined via analysis of X-ray data from the ROSAT All-Sky Survey (see Voges 1993) and statistical measures of clustering from optical images. The X-ray data confirmed that the majority of the radio sources were associated with $X$-ray emission. We also noted a probable difference between FR I and FR II environments, with the FR I sources more frequently associated with extended X-ray emission and optical overdensities of galaxies. Thus, the FR I sources did appear to be associated with poor clusters, while the FR II sources were more isolated.

In this paper, we report on the use of multifiber spectroscopy to further investigate the environments of powerful nearby radio galaxies that are not members of classical Abell clusters. The optical analysis 
employed in Paper I included no velocity information and was instead based on the two-point spatial correlation function $B_{\mathrm{gg}}$. Velocity measurements are therefore necessary to confirm the presence of poor clusters around FR I radio galaxies and further explore the environments of radio galaxies that appear to reside in very sparse regions of galaxies. The velocities are used to evaluate velocity dispersions in the identified poor clusters and estimate system masses under the assumption of virial equilibrium. In some cases, the obtained velocities also allow an investigation of potential substructure within the identified clusters and the relationship of the radio sources to such substructure. The goals are (1) to confirm the existence of poor clusters identified through the presence of powerful radio galaxies and (2) to better understand the relationship between the characteristics of the radio galaxies and their local environments.

The observations and reductions will be discussed briefly in $\S 2$, followed by the computation of quantities to characterize their environments $(\S 3)$. A discussion of the results is presented in $\S 4$, followed by a brief summary of the conclusions. Throughout this paper, we have adopted $H_{0}=75 \mathrm{~km} \mathrm{~s}^{-1}$ $\mathrm{Mpc}^{-1}$ and $q_{0}=0.1$ in all calculations that require such factors.

\section{DATA}

\subsection{The Sample}

The complete sample is composed of 25 radio galaxies drawn from the 3CRR (Laing, Riley, \& Longair 1983) and Wall \& Peacock (1985) catalogs, plus 24 additional radio galaxies drawn from the B2 catalog (Colla et al. 1970, 1972). The B2 sources are generally of lower radio luminosity but were added because the declination range of that survey made them excellent targets for observation at Kitt Peak. The radio galaxies were required to be nearby $(z<0.06$ for the $3 \mathrm{CRR}$ and Wall \& Peacock galaxies, $z<$ 0.04 for the B2 galaxies) and not members of Abel clusters. We report on a subset of 25 of these radio galaxies in this paper. The remainder were unobservable because of declination limits of the telescope (six sources) and observing time lost because of weather. The full sample of radio galaxies and comments on their properties may be found in Table 1.

TABLE 1 3C AND B2 NON-ABell Cluster Radio GalaXies

\subsection{Observations and Reductions}

Spectra were obtained using the MX Spectrometer on the Steward Observatory's $2.3 \mathrm{~m}$ Bok Telescope. MX utilizes $2^{n}$ fibers on mechanical probes to obtain spectra of up to 32 targets at once, plus 
sky spectra from 30 fibers "piggybacked" to target-spectra probes. The field of view of the telescope with MX is $45^{\prime}$, which matches nicely with the angular sizes of the poor clusters being studied. In 18 of the 25 systems being studied, this corresponds to a linear size of $1 \mathrm{Mpc}$ or greater, while for the nearest system it is $\sim 0.8 \mathrm{Mpc}$. The actual spectrograph is the Steward Observatory Boller \& Chivens spectrograph, which rests on the dome floor. Using a 400 line $\mathrm{mm}^{-1}$ grating, $3300 \AA$ coverage is obtained at a resolution of about $6 \AA$. The wavelength center for the observations was typically around $5400 \AA$, which covered from [O II] $\lambda 3727$ to [S II] $\lambda \lambda 6717,6731$ for the small redshifts of the sample. Additional details on the MX instrument may be found in Hill \& Lesser $(1986,1988)$.

Targets were selected from the Digitized Sky Survey plates and corresponded to galaxies with magnitudes near that of the radio galaxy. In all but one calse (B2 0120+33), the fields around the radio galaxies were sparse enough that all relatively bright gallaxies could be observed through a single pointing of the telescope. The integration time for each pointing was I hr. Upon completion of each science exposure, comparison-lamp arc spectra and continuum fiber flats (used to accurately locate the apertures corresponding to each fiber on the $\mathrm{CCD}$ ) were obtained.

Data reduction was performed following the usual steps. On each night of observations, a sequence of 10 zero-second exposures and a sequence of 10 exposures of a quartz lamp (taken through a diffuser placed below the slit) were taken to create a master bias frame and flat field, respectively. The extracted spectra were wavelength-calibrated using the arc lamp exposures, producing rms errors of under $0.2 \AA$ (determined from $\sim 30$ lines and confirmed via inspection of airglow lines). The 30 sky fibers from each pointing were combined to create a super-sky spectrum (after rejecting any fibers that chanced to lie upon stars or galaxies), which was iteratively subtracted from each spectrum until the [O I] night-sky line at $5577 \AA$ was entirely removed.

\subsection{Inspection of Spectra and Velocity Measurements}

Each spectrum was carefully inspected in IRAF. Artifacts from cosmic rays and bad columns on the $\mathrm{CCD}$ were excised from the spectra, and velocity measurement then followed one of two procedures. First, some of the observed galaxies had spectra including emission lines such as the Balmer lines and forbidden oxygen, nitrogen, and sulfur lines. When such lines were present, velocities and errors were obtained using known laboratory wavelengths of such species. In these calculations, all lines were assigned equal weight in the determination of a galaxy's velocity, and the estimate of the error in this quantity is simply the dispersion of the values obtained for individual lines. A minimum velocity error of $20 \mathrm{~km} \mathrm{~s}^{-1}$ was adopted as an upper limit, based on the uncertainty of our wavelength solutions. For the majority of the galaxies, emission lines were not present or were too weak to be useful in velocity characterization. Radial velocities for such galaxies were determined via Fourier cross-correlation (Tonry \& Davis 1979) using known velocity standards. A set of templates including M31, M32, NGC 3379, NGC 7331, and the brightest galaxies from several nearby clusters were used as the velocity standards, with the cross-correlations using wavelengths between 3700 and $6750 \AA$ (minus small regions centered on the prominent night-sky lines at 5577 and $6300 \AA$ ). Each galaxy spectrum was 
cross-correlated against each template spectrum, and the resulting velocities were $3 \sigma$ clipped. Final velocities were assigned if at least $60 \%$ of the templates produced a consistent velocity with an internal dispersion among the individual measurements that was less than $250 \mathrm{~km} \mathrm{~s}^{-1}$. In general, the proximity of the targets resulted in $R \gtrsim 8$ (see Tonry \& Davis 1979). Velocity errors were assigned using the formula $280(1+R)^{-1}$ (Tonry \& Davis 1979; Hill \& Oegerle 1998), again with a minimum of $20 \mathrm{~km} \mathrm{~s}^{-1}$. Finally, each galaxy velocity was confirmed via visual inspection through identification of major features such as the $4000 \AA$ break and prominent absorption features such as the G band, $\mathrm{Mg}$ II, and Na D.

The relative proximity of the sample meant that velocities for some galaxies in the fields of the radio galaxies had already been measured. Consequently, we obtained velocities from the NASA/IPAC Extragalactic Database (NED) for galaxies within $1 \mathrm{Mpc}$ of the radio galaxy positions. For galaxies in the field of 3 C 31, we have adopted the velocities of Ledlow et al. (1996), since these observations were made using the same telescope and detector.

Table 2 presents the results for all galaxies deemed to be associated with the radio galaxies (see $\S 3$ ). The same information for foreground and background galaxies may be found in Table 3 . If emission lines were present in the spectrum, they are noted in the final column. When such lines were not used in the velocity calculation, they are set off by parentheses.

TABLE 2 3C/B2 Cluster Member Velocities

TABLE 3 ForEground AND BACKGROUND GALAXY VELOCITIES

\section{ANALYSIS}

\subsection{Velocity Completeness}

The primary goals of this study are to confirm the presence of poor clusters around the radio galaxies and better understand the connection of these galaxies to their local environments. For these purposes, the biases inherent in our sampling of galaxy velucities should not be too important. However, it is instructive to examine the completeness of our velocity data. Hence, we evaluate the fraction of galaxies 
with measured velocities as a function of optical magnitude and radial separation from the radio galaxies.

Galaxy identifications and magnitudes are available for 21 of the 25 radio galaxy fields in the Automated Plate Scanner (APS) catalogs (for a description of the scanner and procedure, see Pennington et al. 1993). The other fields are generally too close to the Galactic plane, causing difficulty in star-galaxy segregation. We have obtained identifications and magnitudes for all galaxies located within a projected separation of $1 \mathrm{Mpc}$ of the radio galaxies and used them to evaluate the completeness of our velocity data. Table 4 presents the results, including the limiting magnitude $\left(R_{\mathrm{C}}\right.$, derived from the Palomar " $E$ " plates) below which we have velocity data for all galaxies (including those not associated with the radio galaxies). Information is provided for all galaxies within $1 \mathrm{Mpc}$ of the radio galaxies, in addition to those within 500 and $250 \mathrm{kpc}$. Our velocity information is more complete in the central regions of the radio galaxy fields, as these were more likely to be the target of our spectroscopic observations. For galaxies within $1 \mathrm{Mpc}$ of the radio galaxy, our average limiting magnitude is 15.3 (for comparison, the radio galaxies range from $m_{R}=11.2$ to $m_{R}=14.6$ ). The fainter galaxies with spectra of high enough singal-to-noise ratio to obtain accurate velocity measurements typically have $m_{R} \sim 17$. Within $250 \mathrm{kpc}$ of the radio galaxies, the limiting magnitude has dropped to an average of 16.1. Therefore, we conclude that the spectroscopy in these fields adequately samples the velocity fields in the vicinity of the radio galaxies.

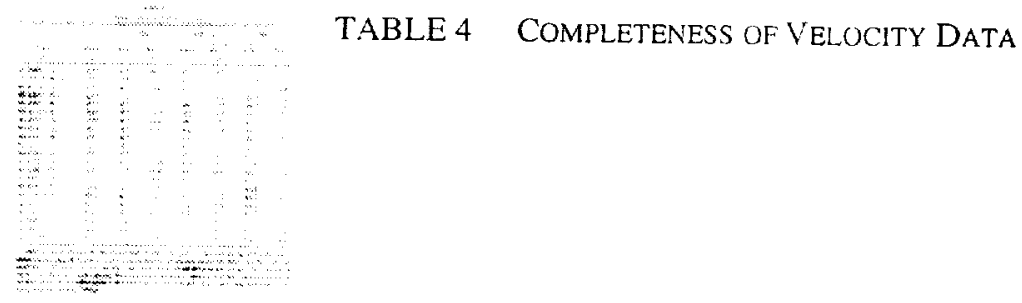

A related issue is the confirmation of the statistical results from Paper I. In that study, counts of the brighter galaxies in the radio galaxy fields were used to evaluate $B_{\mathrm{gg}}$. The velocity data confirm that for three of the six fields in common between these studies (3C 293, 3C 296, and 1615+351; 3C 386 is too close to the Galactic plane to be included in the APS catalog), all of the galaxies used in the $B_{\mathrm{gg}}$ calculations are cluster members. In a fourth ( $3 \mathrm{C} 31$ ), nine out of 10 are confirmed cluster members, a result consistent with the estimate of background gallaxies in the $B_{\mathrm{gg}}$ calculation. The remaining source (3C 305) had seven possible members in the $B_{\mathrm{gg}}$ analysis, of which only three were cluster members. Thus, the statistical evidence for clustering in this radio galaxy field was misleading. However, 3C 305 was shown to not be associated with extended X-ray emission and was therefore a poor candidate for a true cluster system.

\subsection{Derived Quantities: Systemic Velocities, Velocity Dispersions, and Virial Masses}


The systemic velocities and velocity dispersions were calculated using $3 \sigma$ rejection (Yahil \& Vidal 1977). Because of the low number of velocities available, we have adopted the biweight estimators of location and scale $\left(C_{\mathrm{BI}}\right.$ and $S_{\mathrm{BI}}$, respectively) instead of the simple mean and standard deviation (Beers, Flynn, \& Gebhardt 1990). These estimators are less sensitive to the presence of outliers and are consequently better suited to the identification of real systems. The $1 \sigma$ confidence intervals in the biweight scale and location were estimated using the jackknife method (Mosteller \& Tukey 1977, p. 133). In all subsequent discussion, the terms systemic velocity and velocity dispersion will be used for $C_{\mathrm{BI}}$ and $S_{\mathrm{BI}}$. It should be noted that other algorithms exist, for example, the DEDICA method, which uses the data to estimate the underlying probability density and thereby identify clusters and assign probabilities that individual galaxies are members (Pisani 1993).

The results may be found in Table 5. Since the radio galaxies may exist in localized substructures within richer environments or the velocity dispersions might be artificially high should unassociated groups be evaluated together, all quantities have been determined for both a $1 \mathrm{Mpc}$ radius and a $250 \mathrm{kpc}$ radius centered on the radio galaxy. It should be noted that the values for the $250 \mathrm{kpc}$ aperture have not had an additional $3 \sigma$ clipping performed but were simply calculated using the culled lists derived from the $1 \mathrm{Mpc}$ apertures. Figure 1 presents velocity histograms for all of the observed radio galaxy fields, using the $1 \mathrm{Mpc}$ radius. In these figures, a range in velocity of $\pm 2000 \mathrm{~km} \mathrm{~s}^{-1}$ with a bin size of $200 \mathrm{~km} \mathrm{~s}^{-1}$ is shown. Verified cluster members are represented as the shaded portion of the histogram, with foreground and background galaxies unshaded. An overlaid Gaussian with center and dispersion corresponding to the systemic velocity and velocity dispersion of the system is also depicted for each field. Lastly, the arrows denote the locations of the radio galaxies.

\section{TABLE 5 SUMMARY DATA}

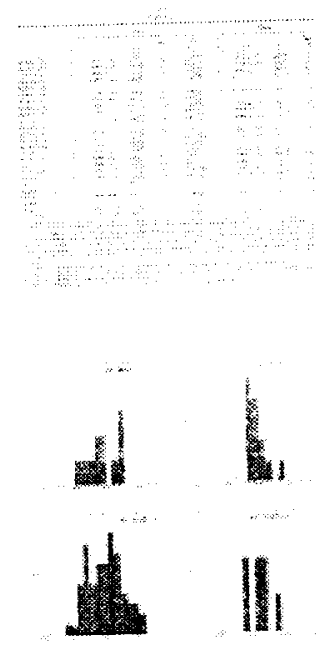

F1G. 1.-Velocity histograms for the 25 sources investigated. For systems consisting of more than one galaxy, a Gaussian with mean and standard deviation corresponding to the derived systemic velocity $\left(C_{B 1}\right)$ and dispersion $\left(S_{B I}\right)$ is also plotted. Unshaded portions of the histograms represent foreground and background galaxies. In the cases of $\mathrm{B} 21322+36$ and $3 \mathrm{C} 305$, the radio galaxy lies just outside of the $3 \sigma$-clipped group. For these two fields, the histogram for the adjacent group is left unshaded, while the radio galaxy is shaded. In all of the histograms, the velocity of the radio galaxy is marked with an arrow. 


$F \equiv=\ldots$


In two cases, the $3 a$ clipping placed the radio galaxy outside of its apparent system. Most notably, B2 $1322+36$ was clipped from a system at $5625 \mathrm{~km} \mathrm{~s}^{-1}$. The overall system appears to consist of nine members, with six of the galaxies tightly grouped in velocity space. These six galaxies produce a velocity dispersion of only $30 \mathrm{~km} \mathrm{~s}^{-1}$, placing the radio galaxy velocity of $5210 \mathrm{~km} \mathrm{~s}^{-1}$ outside of the identified group. Other methods would likely include all of these galaxies in the identified system; in fact, the system has also been noted in prior group studies (e.g., White et al. 1999) that included the radio galaxy among its members. Simple use of the standard mean and deviation (including $3 \sigma$ clipping) produces a single nine-galaxy system with $c z=5614 \mathrm{~km} \mathrm{~s}^{-1}$ and $\sigma=200 \mathrm{~km} \mathrm{~s}^{-1}$. We therefore conclude that this is likely a loose system dominated by a group of galaxies strongly clustered in velocity space. The other radio galaxy that was clipped from a nearby system was $3 \mathrm{C} 305$. It lies near a pair of galaxies at approximately the same velocity.

The distributions and velocities of the galaxies were also used to estimate the masses of the potential clusters, under the assumption of virial equilibrium. The virial masses were calculated using the standard equation:

$$
M=\frac{3 \pi}{G} \sigma^{2}\left(\frac{1}{r}\right)^{-1}
$$

where the harmonic mean radius is defined as

$$
\left(\frac{1}{r}\right)^{-1}=D_{L} N_{p}\left(\sum_{i<j}^{N} \frac{1}{\theta_{j}}\right)^{-1}
$$

and $N_{p}$ is the number of galaxy pairs $[N(N-1) / 2], \theta_{i j}$ is the angular separation of each $i j$ pair, $D_{L}$ is the distance of the cluster, and $\sigma$ is the line-of-sight velocity dispersion. Errors in the velocity dispersions were propagated into the mass estimates. The resulting values were converted into units of solar masses and may be found in Table 5. We have not applied any "surface-term correction" to the mass estimates (The \& White 1986), because this correction requires knowledge of the mass-density profiles. The mass-density profile would be obtained from some assumption of how well the galaxies trace the mass and how well the overall galaxy distribution is sampled. In addition, should substructure be present in the clusters (see $\$ 3.3$ ), application of the virial theorem is obviously inaccurate. These factors tend to produce an overestimate of the true mass. Thus, the formal errors are larger than those quoted in Table 5 . Given these caveats, the masses are generally typical of groups and poor clusters, ranging up to $\sim 3 \times$ $10^{14} M_{\Theta}$.

\subsection{Simple Dynamical Analysis}

A potentially important factor in assessing the environment of the radio galaxies is their location 
within any surrounding clusters. Specifically, with the velocity data in hand we may ask whether the radio galaxies appear to be at rest within the clusters or have some relative motion. Relative motion between the host galaxy and the intracluster medium is required in order to explain certain radio morphologies, such as the bending of tails. In particular, narrow-angle-tail (NAT) morphologies require substantial relative motion. However, in general we might expect the radio galaxies to be nearly at rest, as they are often associated with the most massive elliptical galaxies in their fields.

Using the systemic velocities and errors along with the velocities and errors for the radio galaxies, the significance at which the radio galaxy velocities differed from those of their host clusters was determined. Most of the radio galaxies were shown to lie at the velocity centers of their systems ( 15 of 25 within $1 \sigma$, including those systems with three or fewer velocities). Only three of the radio galaxies (B2 $0222+36, B 21321+31$, and B2 1322+36) appear to have velocities that differ by more than $2 \sigma$ from the velocities of their assumed systems. The result is similar when assessing only those galaxies within $250 \mathrm{kpc}$ of the radio sources (B2 $0222+36$, B2 $1322+36$, and $1615+351$ had greater than $2 \sigma$ velocity offsets). It is perhaps not surprising that these three radio sources are the only ones in the sample that are not the brightest galaxies in their respective fields. However, the low number of velocities for most of the radio galaxy fields in the sample prevent any strong conclusions (see Table 5 for numbers of spectroscopically identified velocities).

Several of the identified groups have fairly large numbers of measured velocities. Zabludoff \& Mulchaey (1998b) investigated six poor groups with large numbers of measured velocities $\left(N_{\text {gal }} \geq 30\right)$ to evaluate hierarchical evolution. Using the $\Delta$-test (Dressler \& Shectman 1988), they confirmed that evidence for substructure was present in two of the poor groups, arguing that these systems evolve hierarchically in much the same way as richer systems such as Abell clusters. Because the presence of substructure has been shown to be linked to radio activity in tailed radio galaxies (Gómez et al. 1997; Bliton et al. 1998; Pinkney et al. 2000), we have applied substructure tests to those groups with more than 20 velocities: B2 $0120+33$ (65 velocities), B2 1621+38 (24 velocities), 3C 31 (52 velocities), 3C 296 (21 velocities), and $1615+351$ (38 velocities).

A battery of statistical tests for substructure were performed for a complete listing of the tests performed, see Pinkney et al. 1996, hereafter PRBB). These tests include a number of normality tests (i.e., comparison of the distribution of velocities with a normal distribution; see the "ROSTAT" routines of Beers, Flynn, \& Gebhardt 1990), tests for substructure in spatial distribution (right ascension and declination), and tests combining both velocity and spatial information. The significance of any detected substructure for the two- and three-dimensional tests was quantified by comparison against 1000 Monte Carlo shuffles of the actual data. Thus, each statistic calculated using the real data was compared with 1000 Monte Carlo simulations to evaluate how likely its value was relative to the null hypothesis of no substructure. For the two-dimensional tests, this null hypothesis is a smooth, azimuthally symmetric distribution of galaxies. A lack of correlation between galaxy position and velocity is the null hypothesis for the three-dimensional tests. The quoted significance level represents how often the test result for the actual data had more substructure than the Monte Carlo shuffles. We adopted a level of $99 \%$ as 
significant (as per the conclusions of PRBB), or fewer than one out of every 100 Monte Carlo simulations showing greater substructure than the real data.

For the most part, evidence for significant substructure was not found. For B2 $0120+33$, the two-dimensional and three-dimensional Lee tests (Lee 1979; Fitchett 1988; PRBB) were each significant at about $95 \%$, and the angular separation test (West, Oemler, \& Dekel 1988) was significant at about 98\%. In total, this represents marginal evidence for the presence of substructure in this cluster. Similarly, $3 \mathrm{C} 31$ had a $\beta$-test (West et al. 1988) that was significant at $98 \%$, and B2 1621+38 had an angular separation test significant at $99 \%$. No other tests were above $90 \%$ significance, as was the case for all tests applied to 3C 296. The strongest evidence for substructure was found in $1615+351$. Its galaxy distribution appears to be elongated, as the Fourier elongation test (PRBB) was significant at well over 99\% confidence. Furthermore, its Lee two-dimensional and three-dimensional tests were also significant at about the $99 \%$ level. While the Fourier test merely identifies an elongated distribution of galaxies, which may or may not be caused by substructure and merging, the Lee tests are insensitive to such distributions. Hence, the combination of these tests argues for the presence of real substructure. The normality tests (based only on velocities) did not show evidence for substructure, although the velocity of the radio galaxy differed from that of the parent cluster by $1.9 \sigma(3.4 \sigma$ from the galaxies within 250 $\mathrm{kpc}$ ).

\section{DISCUSSION}

The majority of the radio galaxies appear to reside in poor clusters or groups. Nineteen of 25 examined fields consisted of at least five galaxies with velocities placing them in a system including the radio galaxy (20 if we include B2 1322+36). In fact, over half of the fields ( 14 of 25 ) had in excess of 10 members, and five had more than 20 members. Two of the radio galaxies, B2 $0120+33$ and $3 \mathrm{C} 31$, were shown to reside in very rich systems, with 65 and 52 confirmed velocities, respectively.

The calculated velocity dispersions and virial masses further indicate that the radio galaxies tend to exist in groups and poor clusters. Figure 2 depicts histograms of the derived velocity dispersions for each the $250 \mathrm{kpc}$ and $1 \mathrm{Mpc}$ counting radii. About a third of the fields have dispersions around $200 \mathrm{~km} \mathrm{~s}^{-1}$, consistent with the values found for nearby groups (e.g., nearly 400 loose groups identified from the Las Campanas Redshift Survey have a median velocity dispersion of $164 \mathrm{~km} \mathrm{~s}^{-1}$ and a median mass of about $2.5 \times 10^{13} M_{6}$; Tucker et al. 2000). Most of the radio-selected groups in the present study have velocity dispersions and masses in excess of these values and more consistent with the velocity dispersions found for groups and poor clusters (see, e.g., Ledlow et al. 1996; Zabludoff \& Mulchaey 1998a). The overall distribution of velocity dispersions is similar to that found by Ledlow et al. (1996), who found a median dispersion of $295 \pm 31 \mathrm{~km} \mathrm{~s}^{-1}$ for a set of optically selected poor clusters. In the present sample, we find a median of $452 \pm 161 \mathrm{~km} \mathrm{~s}^{-1}$ (using only the 20 clusters with five or more velocities, to be consistent with Ledlow et al.). While these values indicate the presence of slightly richer environments around the radio galaxies, it must be noted that Ledlow et al. used a smaller $0.5 \mathrm{Mpc}$ counting radius. The richer among the radio-selected groups have dispersions and masses in line with the poorer Abell clusters. The 
median velocity dispersion of the richness class 0 clusters reported by Zabludoff, Huchra, \& Geller (1990) is around $500 \mathrm{~km} \mathrm{~s}^{-1}$, a value that is exceeded by seven of the radio groups in our sample. The median velucity dispersion for richness class 1 clusters in the same study is about $725 \mathrm{~km} \mathrm{~s}^{-1}$, which is in excess of any of the velocity dispersions for our radio galaxy fields. Thus, the radio galaxies generally reside in structures ranging from groups on up through the poorer Abell clusters.

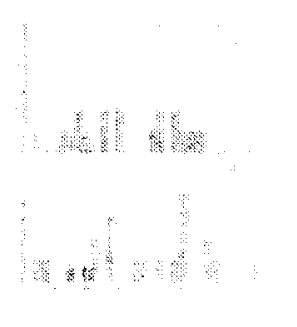

FIG. 2.-Distribution of the derived velocity dispersions for the sample. Top, dispersions calculated within $250 \mathrm{kpc}$ (projected) of the radio galaxy; bottom, those calculated for galaxies within I Mpc.

Galaxy groups have received a great deal of attention in the literature, as their high local densities and low velocity dispersions make them attractive sites for galaxy evolution. It is therefore not surprising that many of the radio galaxy fields have been associated with groups in prior studies. For example, eight of the 25 fields studied in this work were noted by White et al. (1999; "WBL" clusters), and a similar number are reported by Mahdavi et al. (2000; the "RASSCALS"). It is also interesting to note that the radio-galaxy poor clusters are frequently associated with larger scale structures. At least four are associated with the Pisces-Perseus supercluster, and another two are associated with known groups at the periphery of Abell 2197 and 2199. Even with these prior identifications, a number of the radio-selected poor clusters appear to be new. Our data more than double the number of velocities for six of the fields that previously had five or fewer publicly available velocities. The most striking example is 3C 296 , for which we have added 16 cluster velocities to the previously known five.

However, five of the fields produced three or fewer member velocities, indicating that these radio galaxies are almost certainly not in groups or poor clusters. How can these objects be explained? As noted previously, the nature of the radio source is an additional parameter of importance (refer to Table 1). Two of the five (B2 $0207+38$ and B2 $1318+34$ ) are powered by star formation and not an active galactic nucleus (AGN). Optical images show them to be spirals, and their spectra (see Figs. $3 a$ and $3 b$ ) show strong emission of Har. In fact, Poggianti \& Wu (2000) discuss B2 0207+38 as a nearby example of a strong dust-extincted starburst, and Condon et al. (1991) demonstrated that B2 1318+34 is a merger-induced starburst. The remaining three radio galaxies in very poor environments are all $\mathrm{AGNs}$, but they differ from the more typical radio sources of the sample. Both 3C 293 and 3C 305 are galaxy-galaxy merger systems (Heckman et al. 1986; Evans et al. 1999), with strong dust features and signs of disturbance (Martel et al. 1999). Most of the radio emission from 3C 293 is contained within a bright central core, and all of the radio emission from 3C 305 is contained within the optical extent of the galaxy, making the radio morphologies of these sources distinctly different from classical FR I galaxies. They also exhibit evidence of star formation, including large amounts of far-infrared emission, the presence of large amounts of molecular gas (Evans et al. 1999), and emission lines in their optical spectra (see Fig. 3c). The remaining radio galaxy that appears to be fairly isolated is $3 \mathrm{C} 386$. Although 
the arguments are weaker, it has some similarities with 3C 293 and 3C 305. Its radio morphology is that of a relaxed double, with a bright core surrounded by diffuse emission over a large area. Consequently, it is not a classical FR I-type radio source.

FIG. 3.-Representative spectra of radio galaxies: (a) B2 0207+38, a dusty starburst; (b) B2 $1318+34$, a starburst; (c) 3C 305 , a strong emission-line $\mathrm{AGN}$; (d) $1615+351$, a radio galaxy dominated by an old stellar population. The majority of the observed radio galaxies have spectra resembling $(d)$.

An additional three of the radio galaxies have LINER spectra (B2 0222+36, B2 0258+35, and B2 $1422+26$ ). In general, these are also located in poorer environments than the classical FR I radio sources. Both B2 $0258+35$ and B2 $1422+26$ have only five confirmed members, and B2 $0222+36$ resides in a system with 14 confirmed members. However, the local environment of this latter source may be more complicated. It is not the brightest galaxy in its field, and it appears to have a velocity that is offset from the systemic velocity. The small number of velocities prohibits any statistical statements, but this system might be a pair of groups - one that includes the radio galaxy and another that includes the brighter galaxy.

The rest of the radio galaxies generally show classical FR I morphologies, including jets of radio emission that extend past the optical boundaries of the galaxies. Optically, they all have absorption-line spectra indicative of older stellar populations. With the exception of B2 $1322+36$, all of these galaxies have velocity dispersions in excess of $200 \mathrm{~km} \mathrm{~s}^{-1}$, indicating their presence in structures ranging from the size of groups on up through poor Abell clusters, with virial masses from about $2 \times 10^{13}$ to $3 \times 10^{14}$ $M_{6}$. This result is in agreement with the conclusions of Paper I: the presence of an FR I radio source is an excellent indicator of an underlying group or poor cluster. ${ }^{2}$

Two of the FR I radio sources (B2 1621+38 and 1615+351) have NAT morphologies. They are located in two of the richer environments of the sample, and each has associated diffuse X-ray emission (Feretti et al. 1995). Bliton et al. (1998) examined NAT sources drawn from a large sample of Abell clusters and noted that clusters with NATs were more likely than radio-quiet clusters to have substructure. Coalescence of substructures can produce the large relative motions between NAT host galaxies and the intracluster gas necessary to explain the radio morphologies of such sources. For B2 $1621+38$, there is only slight evidence of substructure, a result undoubtedly related to the somewhat limited number of velocities (24) in our study. The situation is improved for $1615+351$, with its 38 velocities producing several significant results (see $\$ 3.3$ ). In addition, the radio galaxy is the second-brightest galaxy in the field, with the brightest being NGC 6107 . This galaxy is also a radio source (Ekers et al. 1978), although it is weaker than 1615+351. Figure 4 plots the galaxy distribution for $1615+351$. The elongation is evident, running from southwest to northeast (which is also consistent with the diffuse X-ray emission; Feretti et al. 1995). A clump of galaxies at lower velocities than the cluster 
center is seen to the northeast. For reference, the radio emission points away from the galaxy toward the northwest. In total, these results indicate that the NATs in our study appear to be found in clusters with substructure, consistent with the findings for NATs in Abell clusters.

FIG. 4.-Galaxy distribution for $16 ! 5+351$. The symbols represent velocity ranges for the galaxies: filled squares, $c z<8410 \mathrm{~km} \mathrm{~s}^{-1}$; filled triangles, $8410 \mathrm{~km} \mathrm{~s}^{-1} \leq c z<8994 \mathrm{~km} \mathrm{~s}^{-1}$; usterisks, $8994 \mathrm{~km} \mathrm{~s}^{-1} \leq \mathrm{cz} \leq 9578 \mathrm{~km} \mathrm{~s}^{-1}$; open triangles, $9578 \mathrm{~km} \mathrm{~s}^{-1}<c z \leq 10,162 \mathrm{~km} \mathrm{~s}^{-1}$; open squates, $c z>10,162 \mathrm{~km} \mathrm{~s}^{-1}$. The radio galaxy (NGC 6109) is at the center of the fjeld and is denoled by a larger symbol. Its velocity is $8979 \mathrm{~km} \mathrm{~s}^{-1}$. The brightest galaxy in the field (NGC 6107 ) has been circled; it is 0.3 mag brighter than the radio galaxy and has a velocity of $9399 \mathrm{~km}$ $s^{-1}$

In general, the stronger radio sources (i.e., after removing star-forming galaxies) can be split into two idealized categories as initially suggested by Heckman et al. (1986). The first class includes the more powerful radio galaxies, with radio emission concentrated in a central core but often including diffuse emission on larger scales. These galaxies frequently have optical emission lines and reside in regions of low galaxy density. They often exhibit signs of disturbance related to recent galaxy-galaxy merging, presumably with at least one of the merger partners a disk galaxy that provides a reservoir of gas to fuel the AGN. The second class are FR I-type sources, which appear more like typical large elliptical galaxies and reside in regions of high galaxy density. Their radio emission extends well past the optical limits of the host galaxies in distinct jetlike structures. Of course, the situation is more complex, as not all radio galaxies can easily be pigeonholed into these two categories.

Unfortunately, our present sample includes no clear FR II galaxies with bright lobes of radio emission. The infrequency of such sources implies that there are very few within the redshift cutoffs we have applied. Such galaxies appear to reside in regions of lower galaxy density, at least for the low redshifts examined in this study. In the future, a comprehensive study of the environments of nearby FR II sources would do much to help understand the possible differences in the environments of FR I and FR II radio galaxies.

The placement of fibers on any galaxies within the fields of the radio galaxies also produced a number of velocities for apparent background groups and clusters. The presence of such systems may be inferred from Table 3. As an example, the observation of B2 1652+39 appears to have netted seven velocities for the cluster Abell 2235. The cluster is presumably located at J2000.0 $\alpha=16^{\mathrm{h}} 54^{\mathrm{m}} 58^{\mathrm{s}}$ and $\delta=40^{\circ} 01^{\prime} 16^{\prime \prime}$ (Abell et al. 1989), which is under $22^{\mathrm{m}}$ from the field center of our observations. Struble \& Rood (1999) report that this cluster has a systemic velocity of $45,300 \mathrm{~km} \mathrm{~s}^{-1}$, determined from three published velocities. From our seven velocities, we find a systemic velocity of $44,454 \pm 120 \mathrm{~km} \mathrm{~s}^{-1}$ with a dispersion of $197_{-85}^{+149} \mathrm{~km} \mathrm{~s}^{-1}$. There is also extended $X$-ray emission associated with this cluster, as indicated by its presence in cluster list generated from the ROSAT All-Sky Survey (identified as RX J1652.6+4011; Böhringer et al. 2000). 
2 As an aside, we note that B2 1652+39, often classified as a BL Lacertae object, resides in an environment consistent with the classical FR I sources of the sample. This is the expected result from FR I-BL Lac unification scenarios.

\section{CONCLUSIONS}

In this paper, we have confirmed that powerful radio galaxies are excellent signposts to the presence of clusters of galaxies. Using a sample of 25 radio galaxies drawn from the 3CRR and B2 catalogs, our velocity measurements determined that $80 \%$ (20/25) consisted of at least five galaxies. Over half of the fields (14/25) had in excess of 10 members, with some having as many as 50 members. The derived velocity dispersions are consistent with those derived for groups and poor clusters. In particular, we note that radio galaxies with classical FR I morphologies seem to reside exclusively in group and poor-cluster environments. The few radio galaxies that exist in regions of particularly low galaxy density are either vigorous star-forming galaxies or complex galaxy-galaxy mergers. These results confirm our prior findings, which were based on statistical measures of galaxy clustering and the presence of extended $\mathrm{X}$-ray emission.

The small sample of radio galaxy fields examined here provides additional clues regarding the formation and evolution of powerful radio galaxies. When the radio galaxies powered by star formation are removed from the sample, there are two general types of radio sources. The first class, which consists of more classical FR I radio galaxies that exhibit clear jets of radio emission, are found in regions of increased galaxy density. These regions have intracluster gas, as inferred through X-ray observations, and this gas can confine the radio plasma emitted by the radio galaxy. The second class of radio galaxies reside in regions of low galaxy density. They often exhibit optical emission lines and evidence of recent merger activity. Thus, environment seems to play a key role in determining the class of the radio galaxy. This picture is clearly an oversimplification, but it generally explains our results. One important further area of study is how more classical FR II sources, with large lobes of bright radio emission, fit into this picture.

The authors thank Dennis Means and Vic Hansen for their assistance during the observations, Jason Pinkney for providing the computer code used in the analysis of possible substructures, and an anonymous referee for comments that substantially improved the focus of the paper. N. A. M. thanks the National Radio Astronomy Observatory for financial assistance in the form of a predoctoral scholarship.

The Digitized Sky Survey was produced at the Space Telescope Science Institute under US government grant NAGW-2166. The images of these surveys are based on photographic data obtained using the Oschin Schmidt telescope on Palomar Mountain and the UK Schmidt Telescope. The plates were processed into the present compressed digital form with the permission of these institutions. The Oschin Schmidt telescope is operated by the California Institute of Technology and Palomar Observatory. The UK Schmidt Telescope was operated by the Royal Observatory Edinburgh, with funding from the UK Science and Engineering Research Council (later the UK Particle Physics and Astronomy Research Council), until 1988 June, and thereafter by the Anglo-Australian Observatory. The blue plates of the Southern Sky Atlas and its equatorial extension (together known as the SERC-J), as 
well as the equatorial red and the second-epoch (red) surveys, were taken with the UK Schmidt.

This research has made use of the APS Catalog of POSS I, which is supported by the National Aeronautics and Space Administration and the University of Minnesota. The APS databases can be accessed at http://aps.umn.edu/. NED is operated by the Jet Propulsion Laboratory, California Institute of Technology, under contract with NASA.

\section{REFERENCES}

- Abell, G. O. 1958, ApJS, 3,211 First citation in article INASA ADS

- Abell, G. O., Corwin, H. G., Jr., \& Olowin, R. P. 1989, ApJS, 70, 1 First citation in article I NASA ADS

- Allington-Smith, J. R., Ellis, R. S., Zirbel, E. L., \& Oemler, A., Jr. 1993, ApJ, 404, 521 First citation in article INASA ADS

- Beers, T. C., Flynn, K., \& Gebhardt, K. 1990, AJ, 100, 32 First citation in article I NASA ADS

- Bliton, M., Rizza, E., Burns, J. O., Owen, F. N., \& Ledlow, M. J. 1998, MNRAS, 301, 609 First citation in article I NASA ADS

- Böhringer, H., et al. 2000, ApJS, 129, 435 First citation in article I Full Text I NASA ADS

- Canosa, C. M., Worrall, D. M., Hardcastle, M. J., \& Birkinshaw, M. 1999, MNRAS, 310, 30 First citation in article I NASA ADS

- Capetti, A., de Ruiter, H. R., Fanti, R., Morganti, R., Parma, P., \& Ulrich, M.-H. 2000, A\&A, 362, 871 First citation in article I NASA ADS

- Colla, G., et al. 1970, A\&AS, 1, 281 First citation in article I NASA ADS

- 1972, A\&AS, 7, l First citation in article INASA ADS

- Condon, J. J., Huang, Z.-P., Yin, Q. F., \& Thuan, T. X. 1991, ApJ, 378, 65 First citation in article 1 NASA ADS

- de Ruiter, H. R., Parma, P., Fanti, C., \& Fanti, R. 1986, A\&AS, 65, 111 First citation in article I NASA ADS

- Dressler, A., \& Shectman, S. A. 1988, AJ, 95, 985 First citation in article I NASA ADS

- Ekers, R. D., Fanti, R., Lari, C., \& Ulrich, M.-H. 1978, A\&A, 69, 253 First citation in article I NASA ADS

- Evans, A. S., Sanders, D. B., Surace, J. A., \& Mazzarellá, J. M. 1999, ApJ, 511, 730 First citation in article / Full Text I NASA ADS

- Fanaroff, B. L., \& Riley, J. M. 1974, MNRAS, 167, 31 P First citation in article INASA ADS

- Feretti, L., Fanti, R., Parma, P., Massaglia, S., Trussoni, E., \& Brinkmann, W. 1995, A\&A, 298. 699 First citation in article I NASA ADS

- Fitchett, M. 1988, MNRAS, 230, 161 First citation in article INASA ADS

- Giovannini, G., Feretti, L., Gregorini, L., \& Parma, P. 1988, A\&A, 199, 73 First citation in article 1 NASA ADS

- Gómez, P. L., Pinkney, J., Burns, J. O., Wang, Q., Owen, F. N., \& Voges, W. 1997, ApJ, 474, 580 First citation in article I Full Text INASA ADS

- Heckman, T. M., Smith, E. P., Baum, S. A., van Breugel, W. J. M., Miley, G. K., Illingworth, G. D., Bothun, G. D., \& Balick, B. 1986, ApJ, 311, 526 First citation in article I NASA ADS

- Hill, J. M., \& Lesser, M. P. 1986, Proc. SPIE, 627, 303 First citation in article I NASA ADS

- 1988, Instrumentation for Ground-based Astronomy: Present and Future, ed. L. B. Robinson (New York: Springer), 233 First citation in article INASA ADS

- Hill, J. M., \& Oegerle, W. R. 1998, AJ, 116, 1529 First citation in article I Full Text I NASA ADS

- Laing, R. A., Riley, J. M., \& Longair, M. S. 1983, MNRAS, 204, 151 First citation in article I 


\section{NASA ADS}

- Laurent-Muehleisen, S. A., Kollgaard, R. I., Feigelson, E. D., Brinkmann, W., \& Siebert, J. 1999, ApJ, 525, 127 First citation in article I Full Text I NASA ADS

- Ledlow, M. J., Loken, C., Burns, J. O., Hill, J. M., \& White, R. A. 1996, AJ, 112, 388 First citation in article I NASA ADS

- Ledlow, M. J., \& Owen, F. N. 1995, AJ, 109,853 First citation in article I NASA ADS

- 1996, AJ, 112, 9 First citation in article INASA ADS

- Lee, K. L. 1979, J. Am. Stat. Assoc., 74, 708 First citation in article

- Liu, F. K., \& Xie, G. Z. 1992, A\&AS, 95, 249 First citation in article INASA ADS

- Longair, M. S., \& Seldner, M. 1979, MNRAS, 189, 433 First citation in article I NASA ADS

- Mahdavi, A., Böhringer, H., Geller, M. J., \& Ramella, M. 2000, ApJ, 534, 114 First citation in article I Full Text I NASA ADS

- Martel, A. R., et al. 1999, ApJS, 122, 81 First citation in article I Full Text I NASA ADS

- Miller, N. A., Owen, F. N., Burns, J. O., Ledlow, M. J., \& Voges, W. 1999, AJ, 118, 1988 (Paper I) First citation in article I Full Text I NASA ADS

- Mosteller, F., \& Tukey, J. W. 1977, Data Analysis and Regression (Reading, MA: Addison-Wesley) First citation in article ( NASA ADS

- O’Dea, C. P., \& Owen, F. N. 1985, AJ, 90, 927 First citation in article INASA ADS

- Owen, F. N., \& Laing, R. A. 1989, MNRAS, 238, 357 First citation in article I NASA ADS

- Owen, F. N., \& White, R. A. 1991, MNRAS, 249, 164 First citation in article I NASA ADS

- Parma, P., de Ruiter, H. R., Fanti, C., \& Fanti, R. 1986, A\&AS, 64, 135 First citation in article NASA ADS

- Pennington, R. L., Humphreys, R. M., Odewahn, S. C., Zumach, W., \& Thurmes, P. M. 1993, PASP, 105, 521 First citation in article I NASA ADS

- Pinkney, J., Burns, J. O., Ledlow, M. J., Gómez, P. L., \& Hill, J. M. 2000, AJ, 120, 2269 First citation in article / Full Text I NASA ADS

- Pinkney, J., Roettiger, K., Burns, J. O., \& Bird, C. M. 1996, ApJS, 104, 1 (PRBB) First citation in article I NASA ADS

- Pisani, A. 1993, MNRAS, 265, 706 First citation in article I NASA ADS

- Poggianti, B. M., \& Wu, H. 2000, ApJ, 529, 157 First citation in article I Full Text I NASA ADS

- Prestage, R. M., \& Peacock, J. A. 1988, MNRAS, 230, 131 (erratum 236, 959 [1989]) First citation in article I NASA ADS

- Struble, M. F., \& Rood, H. J. 1999, ApJS, 125, 35 First citation in article I Full Text I NASA ADS

- The, L. S., \& White, S. D. M. 1986, AJ, 92, 1248 First citation in article I NASA ADS

- Tonry, J., \& Davis, M. 1979, AJ, 84, 1511 First citation in article I NASA ADS

- Tucker, D. L., et al. 2000, ApJS, 130, 237 First citation in article I Full Text I NASA ADS

- Venturi, T., Giovannini, G., Feretti, L., Comoretto, G., \& Wehrle, A. E. 1993, ApJ, 408, 81 First citation in article INASA ADS

- Voges, W. 1993, Adv. Space Res., 13(12), 39l First citation in article I NASA ADS

- Wall, J. V., \& Peacock, J. A. 1985, MNRAS, 216, 173 First citation in article I NASA ADS

- West, M. J., Oemler, A., Jr., \& Dekel, A. 1988, ApJ, 327, I First citation in article INASA ADS

- White, R. A., Bliton, M., Bhavsar, S. P., Bornmann, P., Burns, J. O., Ledlow, M. J., \& Loken, C. 1999, AJ, 118, 2014 First citation in article I Full Text I NASA ADS

- Yahil, A., \& Vidal, N. V. 1977, ApJ, 214, 347 First citation in article INASA ADS

- Zabludoff, A. I., Huchra, J. P., \& Geller, M. J. 1990, ApJS, 74, 1 (erratum 75, 629 [1991]) First citation in article I NASA ADS

- Zabludoff, A. I., \& Mulchaey, J. S. 1998a, ApJ, 496, 39 First citation in article I Full Text INASA $\mathrm{ADS}$ 
- $-1998 \mathrm{~b}, \mathrm{ApJ}, 498$, L5 First citation in article / Full Text I NASA ADS 